\title{
Diagramas e provas 1
}

\author{
Abel Lassalle Casanave \\ UFSM / CNPq abellassallecasanave@smail.ufsm.br \\ Bruno Vaz \\ Doutorando PUC-RJ / Bolsista de Doutorado CNPq \\ Sérgio Schultz \\ Doutorando PUC-RJ / Bolsista de Doutorado CNPq
}

resumo A concepção padrão de prova é uma concepção lingüística de prova. No entanto, literatura recente reivindica a legitimidade de provas heterogêneas, isto é, que incorporem recursos visuais ou gráficos. Tal reivindicação implica em um melhor exame da distinção entre representação lingüística e representação gráfica ou visual; concomitantemente, ela também comporta uma análise da dualidade entre discursivo e intuitivo em filosofia da lógica e da matemática. Neste breve artigo examinamos dois exemplos canônicos de provas heterogêneas, salientando, no entanto, seu caráter discursivo. Em uma também breve secção final introduzimos com caráter conjetural o conceito leibniciano de representação ecthetica com vistas a repensar a distinção entre lingüístico e gráfico.

palavras-chave provas heterogêneas; representação lingüística; representação gráfica.

Además, los signos son tanto más útiles cuanto más expresan el concepto de la cosa significada en forma tal que no sólo pueden servir para la representación sino también para el razonamiento. (Leibniz, 1982, p. 189)

Signos são para Leibniz, entre outros, as palavras, as figuras geométricas, as letras, os hieróglifos, os diagramas em geral, as notações aritméticas e algébricas, assim como a musical, os mapas, as tabelas, etc. Tudo isso representa, mas por vezes serve também para o raciocínio, para inferir e provar. Naturalmente, bons leitores lêem nos escritos de Leibniz acerca da distinção entre representação lingüística e representação gráfica, assim como Recebido em 11 de março de 2009. Aceito em 19 de agosto de 2009. doispontos, Curitiba, São Carlos, vol. 6, n. 2, p.13-25, outubro, 2009 
sobre a distinção entre provas sentenciais e provas heterogêneas ou mistas, isto é, que incorporam recursos gráficos além de lingüísticos, visualização além de discursividade.

Um par de exemplos. Com linhas representamos retas, círculos e outras figuras que servem para o raciocínio geométrico, ao menos em uma prova à maneira dos Elementos de Euclides. Com círculos representamos conceitos que servem para o raciocínio lógico em uma prova por diagramas de Venn. As provas são heterogêneas: envolvem, além de sentenças de uma linguagem, os correspondentes diagramas. Por certo, "prova heterogênea" era uma expressão até pouco tempo atrás não autorizada pelo uso: provas são sentenciais, excluindo por definição recursos gráficos.

Com efeito, uma das conseqüências do debate fundacional, de fins do século XIX e início do século XX, foi uma revisão do conceito de prova. No lugar de um conceito mais amplo de prova, que permitia, por exemplo, a utilização de diagramas e o recurso à suposta intuição geométrica, surgiu, sob a influência de Frege, Russell e Hilbert, entre outros, a concepção exclusivamente sentencial de prova que culmina na noção de prova formal. Uma prova formal é uma seqüência de fórmulas tal que cada uma delas, ou bem é um axioma, ou bem se segue de fórmulas anteriores na seqüência por meio de uma regra de inferência. A idéia básica por trás deste conceito de prova é a de que provas, para serem legítimas, têm de ser rigorosas, sendo o rigor identificado com a explicitação de axiomas e regras de inferência. Obviamente, provas que utilizem recursos diagramáticos não seriam rigorosas, portanto, seriam ilegítimas; obviamente, as únicas provas legítimas seriam as provas formais, nas quais os diagramas não desempenhariam nenhum papel fora o papel ilustrativo.

Retornemos aos exemplos acima. Uma autêntica prova geométrica exige então explicitar todos os axiomas (os lógicos e os da teoria de que se trate), e regras de inferência, de forma tal que nunca o diagrama venha justificar qualquer passo da seqüência que constitui a prova. ${ }^{2}$ Uma autêntica prova de uma forma válida de inferência, como um silogismo, exige uma seqüência semelhante a partir dos axiomas lógicos e das regras de inferência explicitados.

Ora, nas últimas décadas do século $\mathrm{XX}$ as provas diagramáticas voltaram ser defendidas como sendo tão legítimas quanto as provas sentenciais, formais em particular. No entanto, a legitimidade das provas 
diagramáticas, como observa Seoane (2007), não estaria associada ao uso de regras de inferência lógica, como ocorre em provas sentenciais. Em outras palavras, o uso de diagramas substitui, ao menos em parte, o uso de regras de inferência. Assim, algumas informações que em provas sentenciais precisam ser inferidas de sentenças anteriores, nas provas diagramáticas podem ser lidas ou extraídas diretamente dos diagramas e, nesse sentido, obtidas 'de graça' a partir deles. O fato das informações que obtemos 'de graça' no decorrer de uma prova com diagramas não serem justificadas, seja por um axioma, seja por uma regra de inferência, não implicaria que essas informações não admitam outra forma de justificação.

O que justifica que possamos ler ou extrair, 'de graça', certas informações a partir dos diagramas? Autores como Barwise, Etchemendy e Shimojima - que estão pensando essencialmente na inferência lógica e não nas provas geométricas - defendem que aqueles passos em provas diagramáticas que não podem ser justificados logicamente são justificados mediante visualização. Deste modo, apesar de que a relação entre os diagramas e as relações conceituais representadas é convencional, nós literalmente visualizamos no diagrama que uma determinada informação pode ser assumida legitimamente. Portanto, provas diagramáticas seriam heterogêneas, pois envolveriam a associação da validade de certos passos propriamente discursivos com fatos visualizados acerca dos diagramas. Esta concepção parece envolver a idéia de que os diagramas atuam, tanto como signos, quanto como meros objetos físicos visualizáveis que instanciam certas propriedades geométricas ou topológicas. Provaríamos resultados sobre os conceitos representados pelos signos por meio da visualização - da intuição empírica - dos objetos físicos que são tokens daqueles signos. Desse modo, o uso de regras de inferência lógica seria substituído pela visualização dos diagramas, e não, por exemplo, pelas regras de uso ou de desenho deles.

Em dois brilhantes estudos sobre a geometria de Euclides, K. Manders propõe uma interpretação das provas euclidianas como constituídas por uma parte textual (definições, postulados, noções comuns e proposições previamente demonstradas) e uma parte gráfica, que contribuem de maneira diferente no curso da demonstração. Uma prova euclidiana consiste em uma seqüência de declarações (claims) na parte textual e uma seqüência de transformações (entries) do diagrama. Ora, algumas dessas 
declarações se justificam apenas pelo recurso ao diagrama, sendo essas provas, portanto, heterogêneas. ${ }^{3}$ Com vistas a determinar quais informações podem legitimamente ser extraídas ou lidas do diagrama, Manders introduz uma distinção entre o que ele denomina aspectos exatos e aspectos co-exatos dos diagramas. ${ }^{4}$ Com isso é possível discriminar quando um passo pode ser justificado pelo recurso ao diagrama, e quando não.

Em que sentido se fala de visualização em ambos os casos? No que segue examinaremos provas geométricas e provas com diagramas de Venn com vistas a melhor qualificar a distinção entre representação lingüística e gráfica, a que está por detrás de falar de visualização, no segundo caso, e discursividade, no primeiro. E em uma breve seção final sugeriremos compreender a heterogeneidade em questão como heterogeneidade de sistemas lingüísticos de representação.

\section{Diagramas geométricos}

Em que consiste uma prova euclidiana? Por um lado, como dissemos, temos uma seqüência de "declarações textuais"; pelo outro, uma seqüência de transformações de um diagrama, ou "entradas diagramáticas”. As declarações textuais determinam, tanto a aparência inicial do diagrama, quanto suas sucessivas transformações; porém, nem toda declaração implica uma transformação do diagrama. Para além da especificação inicial, as entradas textuais que introduzem modificações no diagrama incluem o recurso, seja aos postulados, seja às proposições de construção, ou problemas. Além disso, o texto inclui declarações com base em definições, ou nas chamadas noções comuns, ou ainda em teoremas previamente demonstrados. Porém, em certos casos, uma afirmação no texto se justifica utilizando informações que "decorrem" somente do diagrama. Em outras palavras: o passo da prova não é discursivo, mas diagramático, visual, pois envolve uma informação que apenas o diagrama fornece. Dada esta heterogeneidade, haveria o recurso supostamente ilegítimo aos diagramas nas demonstrações dos Elementos. E esse recurso intuitivo deveria ser eliminado pela prova sentencial "puramente" discursiva, como fez Hilbert em Os fundamentos da geometria. 
Exemplifiquemos com uma objeção canônica à prova do teorema I.1: dado um segmento de reta, construir um triângulo de lado igual ao segmento dado. A uma declaração textual inicial (a chamada ekthesis) corresponde uma "entrada" diagramática associada: seja AB um segmento de linha reta:

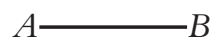

Sucessivas declarações textuais modificam a aparência inicial do diagrama (novas entradas diagramáticas): pelo Postulado 3, traçando um círculo com centro em A e extremo em B, primeiro; e, depois, traçando um círculo com centro em B e extremo em A.

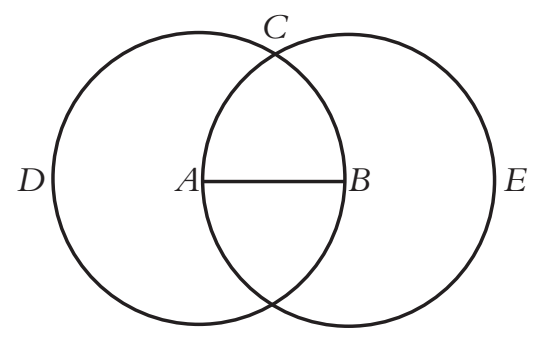

Finalmente, duas aplicações do postulado 1 nos permitem unir o ponto $\mathrm{C}$ determinado pela interseção dos dois círculos com A e B.

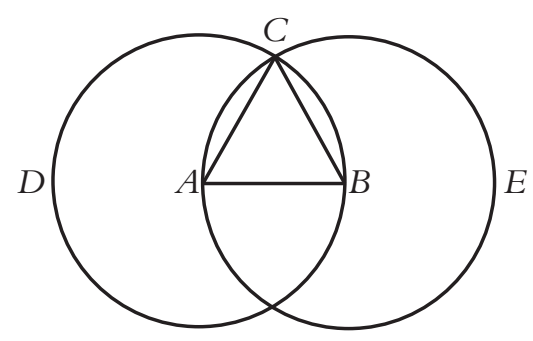


$\mathrm{O}$ problema não consiste em justificar as retas entre os pontos $\mathrm{A}$ e $\mathrm{C}$ ou $\mathrm{B}$ e C, dado que isto é permitido pelo Postulado 1, mas sim a (falta de) justificação para a existência do ponto C. Ora, o diagrama contribui certamente para a prova, porém essa contribuição não é irrestrita.

Por exemplo, não podemos dizer que vemos círculos ou retas no diagrama: o que vemos, propriamente falando, são curvas, algumas das quais são "abertas" (uma vez que não encerram espaço) e outras são fechadas. Esses são os aspectos exatos, que não podem ser encontrados no diagrama porque esse, por mais bem desenhado que seja, nunca instanciará retas ou círculos perfeitos. Dito de outro modo, os chamados aspectos exatos são sensíveis a deformações do diagrama, mas os aspectos chamados co-exatos, por sua vez, são insensíveis a uma ampla gama de deformações: que uma região seja parte de outra, ou que seja limitada por um segmento, são exemplos de relações que são mostradas pelo diagrama em qualquer diagramação adequada, sendo, portanto, informações que podem ser lidas ou extraídas legitimamente do diagrama.

Com efeito, segundo Manders, o recurso à aparência do diagrama deve ser considerado legítimo quando se trata de um aspecto insensível a deformações. Ora, não pode haver diagramação dos dois círculos que respeite minimamente as estipulações do caso e que, ao mesmo tempo, não mostre os dois pontos de intersecção dos círculos. $\mathrm{O}$ famigerado ponto $\mathrm{C}$ aparece em qualquer diagramação minimamente adequada: ele é o primeiro exemplo nos Elementos de um aspecto co-exato lido diretamente do diagrama produzido. Dessa perspectiva, a existência do ponto C deixa de ser polêmica: forma parte da disciplina de produção dos diagramas. ${ }^{5}$ A interseção dos círculos, no entanto, é apenas um dos tantos aspectos co-exatos que decorrem de declarações textuais e das conseqüentes modificações por elas engendradas nos diagramas. $\mathrm{O}$ diagrama nos permite reconhecer regiões, nos permite reconhecer que uma região seja parte de outra ou que um ponto seja interior ou exterior a uma região, porém que os diagramas representem círculos, triângulos ou o centro de um círculo - para seguir nosso exemplo - isso somente o texto permite afirmar.

A continuação da demonstração fornece exemplos interessantes do que não pode ser lido do diagrama: que os segmentos $\mathrm{AC}$ e $\mathrm{AB}$ sejam retas; que, além disso, sejam iguais. Com efeito, que $A C$ e $A B$ sejam 
retas - um aspecto exato do diagrama - somente podemos sabê-lo pelo texto; que sejam qualitativamente iguais - outro aspecto exato decorre textualmente argumentando que são raios do mesmo círculo. $\mathrm{O}$ mesmo para $\mathrm{AB}$ e $\mathrm{BC}$. E, finalmente, que $\mathrm{AC}$ e $\mathrm{BC}$ sejam iguais decorre também textualmente de uma noção comum: coisas iguais a uma terceira são iguais entre si. Logo, a demonstração está concluída. (A justificação da igualdade "qualitativa" de dois segmentos passa mediata ou imediatamente pela noção de círculo, a saber, dois segmentos são iguais se são raios do mesmo círculo.)

Como podemos ver, a parte diagramática da demonstração é limitada sobremaneira por sua parte textual, de modo que a alegação de que o uso de diagramas torna uma demonstração deficiente já não pode ser sustentada tout court. Há, com efeito, padrões também rigorosos de leitura do diagrama. Por exemplo, se há uma diagramação alternativa que não apresente a relação em questão, ela já não poderá ser lida do diagrama, pois nesse caso o resultado alcançado perderia sua generalidade. Cabe ressaltar também que há um limite para as deformações que são aceitáveis: o diagrama de um círculo, por exemplo, pode se assemelhar a uma elipse; mas não pode ser tal que não se consiga identificar nele uma região interna e uma externa.

\section{Diagramas de Venn}

Na prova da validade de um silogismo, consideramos se uma conclusão se segue ou não de modo logicamente válido a partir de um par de premissas. Tanto as premissas, quanto a conclusão, são proposições categóricas da forma: "Todo S é P", "Nenhum S é P", “Algum S é P" e "Algum S não é $\mathrm{P}$ ”. Os diagramas, por sua vez, são compostos por três círculos ou elipses parcialmente sobrepostos, de modo a apresentar regiões exclusivas de cada um dos círculos, regiões de intersecção, dois a dois, entre eles, e uma região de intersecção dos três, como no diagrama abaixo: 




Como é bem conhecido, a prova consiste em diagramar as premissas para ver se resulta diagramada "de graça” a conclusão. Há, portanto, duas classes de entradas no diagrama, ou "marcações": hachuras de regiões, que representam relações conceituais de subordinação e exclusão; e cruzes em regiões, que representam relações conceituais de subordinação e exclusão parciais. Por exemplo, provemos a validade do silogismo Ferio ("Nenhum $\mathrm{M}$ é P, Algum S é M; logo Algum S não é P"). À premissa "Nenhum M é P" (exclusão conceitual de $\mathrm{M}$ e $\mathrm{P}$ ) corresponde uma entrada diagramática, isto é, uma marcação, a saber, a hachura da intersecção de M com P, como diagramado a seguir:

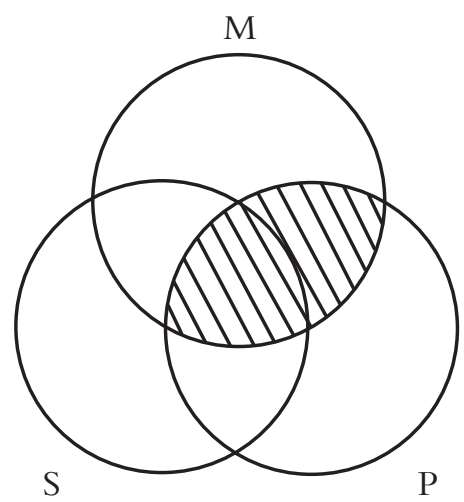

Ora, podemos afirmar que com essa marcação foram introduzidos aspectos co-exatos, a saber, por exemplo, que uma cruz não pode ser marcada na sobreposição dos três círculos.. Justamente, a seguir, há uma 
nova entrada (marcar uma cruz na área comum a $\mathrm{S}$ e $\mathrm{M}$ ) em função de uma segunda premissa, isso é, da proposição "Algum S é M" (subordinação parcial entre $\mathrm{S}$ e $\mathrm{M}$ ). Como hachuramos toda a área comum a $\mathrm{M}$ e $\mathrm{P}$, que inclui a área comum a $\mathrm{S}, \mathrm{P}$ e $\mathrm{M}$, somente podemos fazer a marcação na área comum apenas a $\mathrm{S}$ e $\mathrm{M}$, de acordo com a disciplina diagramática requerida para a produção destes diagramas. Conseqüentemente, obtemos o diagrama abaixo:

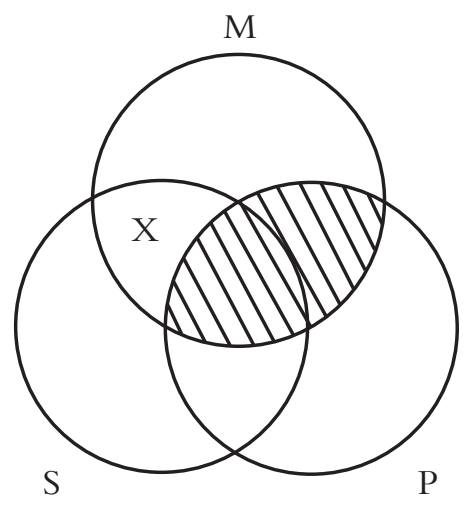

Nesse diagrama há uma cruz marcada na área comum a $\mathrm{S}$ e $\mathrm{M}$ e que não é comum a P. Logo, também está diagramada "Algum S não é P" (exclusão parcial entre $\mathrm{S}$ e P), informação que é lida ou extraída do diagrama como resultado da relação das duas entradas diagramáticas anteriores.

De um modo geral, como dissemos na seção anterior, os aspectos coexatos são aqueles que são insensíveis a uma ampla gama de deformações do diagrama e que, devido a isso, podemos extrair informações a partir deles. Esse parece ser o caso quando extraímos do diagrama a marcação correspondente à conclusão, como resultado da inter-relação das marcações introduzidas pelas premissas. Embora constitua um caso menos complexo de demonstração heterogênea, o método de Venn também exige uma disciplina diagramática. Nesse caso ela dirá respeito à habilidade de produzir as marcações do caso. Finalmente, ainda que tenhamos falado de aspectos co-exatos, isto é, de aspectos que dependem da aparência do diagrama, deixaremos para outra oportunidade a questão de se há, e em que medida, um análogo para os chamados aspectos exatos. 


\section{Breve e inconclusivo final}

Os diagramas de Venn não são instâncias das formas ou relações lógicas que representam. Como ocorre com sentenças e palavras, que o diagrama possua certa aparência significa que ele é uma inscrição de um determinado signo que, por sua vez, "representa" esta ou aquela noção ou proposição. O aspecto co-exato segundo o qual, no diagrama acima, a área do círculo $S$ que não é comum a $\mathrm{P}$ contém uma cruz, permite dizer que aquele diagrama é um signo que representa a proposição “Algum S não é P”. Ora, são, por sua vez, as figuras geométricas "instâncias" dos conceitos geométricos correspondentes? Na medida em que são instâncias, não permitem justificar passos da demonstração; na medida em que são signos sujeitos a manipulação regrada sim podem justificar passos de prova. Isso sugere que, em ambos os casos, sejam os diagramas de Euclides ou de Venn, a aparência do diagrama, sua "topologia", diz respeito a sua condição de signos.

Que classe de signos? A reivindicação do pensamento diagramático tem comportado, ao menos em parte, uma dimensão perceptual, especificamente visual, de caráter não-lingüístico. Porém, os diferentes intentos de distinguir representação gráfica de representação lingüística parecem não ser conclusivos. Shimojima (2001) recenseou os principais intentos com vistas a caracterizar a distinção entre representação gráfica e lingüística: a) representação analógica vs. digital; b) representação com estrutura não-seqüencial vs. representação com estrutura seqüencial; c) representações que não utilizam símbolos para propriedades ou relações para expressar propriedades ou relações do representado vs. representações que utilizam tais símbolos; d) representações mais homomórficas vs. representações menos homomórficas do representado; e) representações cuja limitação expressiva consiste em que não podem apresentar uma informação sem deixar de apresentar outras vs. representações que não padecem dessa limitação; f) representações estruturadas de forma tal que devem obedecer a restrições extrínsecas e intrínsecas vs. representações que somente devem obedecer a restrições extrínsecas. Finalmente, a proposta de Shimojima; g) representações sujeitas a restrições nomológicas vs. representações sem restrições nomológicas. Sem entrar em maiores detalhes, a conclusão parece ser que a distinção entre representação gráfica e representação lingüística é, gradual. 
Para nós, parte do problema passa por revisar noções como linguagem e escritura. Com efeito, sob a rubrica "linguagem" entram, tanto as linguagens naturais - que intervêm crucialmente nas provas euclidianas -, quanto as linguagens formais - que intervêm crucialmente no modo formal (algébrico) de provar. E sob a rubrica "escritura" entram, tanto as escrituras fonéticas usuais, quanto as logo-gráficas como a chinesa e as escrituras analíticas próprias das linguagens formais. Ora, já se tem afirmado, por exemplo, o caráter gráfico da notação bi-dimensional fregeana. ${ }^{6}$ E o que dizer da quantificação ramificada que permite exprimir relações lógicas que a notação unidimensional usual não permite? Por esse caminho, poderíamos concluir que as escrituras analíticas são, na verdade, representações gráficas. Ou simplesmente exemplificar que a distinção entre representação gráfica e lingüística é gradual.

Ora, começamos esse artigo com uma epígrafe de Leibniz, e com uma referência ao Leibniz o concluímos, lembrando a noção de representação ecthetica, isto é, de uma representação que, mais que exibir ou mostrar nos signos ou caracteres "relações estruturais" do representado, presentifica esse último. Esse tipo de representação é ilustrado principalmente, porém não somente, pelas escrituras analíticas, como a da aritmética e da álgebra, que, além de representar, servem para o raciocínio, inclusive no sentido de cálculo. Conjecturamos que, em casos como os diagramas de Venn, se trata de escrituras que partilham o modo de representação ecthetico, escrituras vinculadas com o cálculo e com o chamado pensamento simbólico. Conjecturamos também que em casos como os das provas geométricas euclidianas, coexiste o componente de pensamento verbal associado com a escritura usual (e dissociado do cálculo) com a dimensão ecthetica da escritura de figuras que também forma parte da linguagem da prova. Não tentaremos provar ambas as conjecturas. Por enquanto.

1 Os autores agradecem ao CNPq pelo financiamento deste trabalho através dos projetos 306044/2007-2 e 475534/2008-5.

${ }^{2}$ Sob esta perspectiva, nem sequer uma prova dos Fundamentos da Geometria de Hilbert seria legítima: embora sentencial no sentido dos diagramas somente servirem aos fins de ilustração, Hilbert não explicitou a "lógica subjacente". 


\section{4}

3 MANDERS (2008a, p. 68).

${ }^{4}$ MANDERS (2008a, p. 69). O autor por vezes se refere também a "condições" exatas e coexatas, ou ainda "passos" ou "características" exatos e co-exatos. Por razões de uniformidade, nos referiremos a eles como "aspectos" exatos e co-exatos.

5 MANDERS (2008b, p. 89) denomina disciplina diagramática à habilidade de produzir diagramas adequados, ainda que imperfeitos.

${ }^{6}$ Veja-se, por exemplo, Greaves (2002).

\section{Referências bibliográficas}

ALLWEIN, G e BARWISE, J. (eds): (1996) Logical Reasoning with Diagrams. Oxford: Oxford University Press.

BARWISE, J. e ETCHEMENDY, J.: (1996a) "Visual Information and Valid Reasoning”. In: ALLWEIN, G e BARWISE, J. (eds): Logical Reasoning with Diagrams. Oxford: Oxford University Press 1996. pp. 3 26.

$$
\text { . (1996b) "Heterogeneous Logic” In: ALLWEIN, G e }
$$

BARWISE, J. (eds): Logical Reasoning with Diagrams. Oxford: Oxford University Press 1996. pp. 179 - 200.

BARWISE, J. e SHIMOJIMA, A. (1995) "Surrogate Reasoning".

Cognitive Studies: Bulletin of the Japanese Cognitive Science Society, 2(4): 726.

EUCLID (1956) The thirteen books of the Elements. New York: Dover.

GREAVES, M.: (2002) The Philosophical Status of Diagrams. Stanford: CSLI Publications.

HILBERT, D. (1980) Foundations of Geometry. La Salle, Ilinois: Open Court.

LEIBNIZ, G. W. (1982) Escritos Filosóficos. Buenos Aires: Editorial Charcas.

MANCOSU, P. (ed.): (2008) The Philosophy of Mathematical Practice. Oxford: Oxford University Press. 
MANDERS, K.: (2008a) “Diagram-Based Geometric Practice”. In:

MANCOSU, P. (ed.): The Philosophy of Mathematical Practice. Oxford:

Oxford University Press 2008. pp. 65-79.

. (2008b) “The Euclidean Diagram”. In: MANCOSU, P.

(ed.): The Philosophy of Mathematical Practice. Oxford: Oxford University Press 2008. pp. 80-133.

SEOANE, J.: (2006) "Representar y demostrar. Observaciones preliminares sobre diagramas", Representaciones, 2 (2), pp. 105-126.

SHIMOJIMA, A. (2001) "The Graphic-Linguistic Distinction". Artificial Intelligence Review, 15: 5-27. 